\title{
Relative Collision Cross Sections of Organic Ions
}

\author{
Stilianos G. Roussis \\ Research Department, Imperial Oil, Sarnia, Ontario, Canada
}

\begin{abstract}
The relative collision cross sections $\sigma_{\mathrm{c}}$ of selected precursor ions that collide with helium at high ion kinetic energies (kiloelectronvolts) have been obtained by monitoring the ion beam attenuation at different collision pressures. A value for the total collision cross section is obtained, which includes contributions from different processes that lead to fragmentation, scattering, charge exchange, charge inversion, and stripping. For $n$-alkanes and $n$ alkylbenzenes the $\sigma_{\mathrm{c}}$ value increases linearly by 0.1 per methylene group. Linear relationships exist between relative collision cross section and ionization cross section $\sigma_{i}$, and polarizability $\alpha$ of the corresponding neutral molecule, but different equations are required for the description of $\sigma_{\mathrm{c}}$ in each chemical series. A simple relationship, which requires only the chemical formula of the precursor ion, has been determined that can predict all relative collision cross sections with an average error of $3.5 \%$. The total relative collision cross section increases with the size of the ion. The experimental results are in agreement with the predictions of the hard-sphere collision theory. The approach can be applied to determine the relative collision cross sections at different ion kinetic energy regimes and collisions with different target gases. (J Am Soc Mass Spectrom 1995, 6, 803-811)
\end{abstract}

Q uantitative relationships between the various precursor ions in tandem mass spectrometry experiments require the knowledge of the relative collision cross sections of the precursor ions. In typical tandem mass spectrometry applications, precursor ions with the same nominal mass undergo collision activation with a target gas. The selected ions may be isomeric or isobaric structures. Although quantitative calibrations with standard compounds for selected precursors can be used, they involve tedious experimental procedures. It is, therefore, desirable to derive information about the relative sensitivities of the various selected ions from correlations with the molecular parameters of the collision system. Such correlations have been developed for 70-eV electron ionization experiments [1, 2]. Similar quantitative relationships, in tandem mass spectrometry experiments that use sector instruments, are more complex to derive. Contrary to the $70-\mathrm{eV}$ gas chromatographymass spectrometry experiments where the assumption is that at the absence of instrumental discrimination, the relative amount of total sample ionization is directly proportional to the relative ionization cross section of the structure [1], in tandem mass spectrometry experiments, a substantial amount of the precursor ion current is lost and does not undergo detection due to

Address reprint requests to Stilianos Roussis, Research Department, Imperial Oil, 453 Christina Street South, P.O. Box 3022, Sarnia, Ontario, Canada N7T 7M1. the competing collision reactions (scattering, etc.). This amount of lost ion current is different for the different precursor ions. The measured total ion current of the product ions is not necessarily equal to the original precursor ion current fraction undergoing collision. Instead of use of the total ion current composed of the ion current from all fragment ions, as used for the determination of the ionization cross sections in $70-\mathrm{eV}$ mass spectrometry experiments [1], the present work makes use of the total ion current of the precursor ion. The total collision cross section is obtained from the slope of the plot of the precursor ion intensities measured at different collision pressures. The information obtained from these experiments is related to the relative sensitivities of the precursor ion structure.

Precursor ions with different masses or chemical structures have different cross sections for collisioninduced dissociation (CID) and ion loss upon collision with a target molecule. Collision-induced dissociation competes with scattering, charge stripping, and charge exchange processes [3]. The probability for occurrence of each of these processes depends on the nature of the binary collision system and the experimental conditions. The total collision cross section of an ion-molecule reaction is a function of the individual cross sections for all competing collision processes. Determination of the cross section for any of the individual collision reactions can provide useful information about the ion structure. The relationship between ion structures and CID cross sections has been studied by van Tilborg and van Thuijl [4]. Structural differen- 
tiation of the various isomers was possible based on their relative CID cross sections.

Due to the uncertainties associated with the various competing collision processes and the experimental difficulties for the measurement of the individual collision cross sections, it is preferable to determine the total collision cross section of the reacting system. Information obtained in that manner includes contributions from all competing reactions. van Houte et al. [5] recently derived structure-related information from the total collision cross sections of ions that collide with a target gas. For $n$-alkanes and $n$-alkanones, they obtained linear relationships between the number of carbon atoms and the total collision cross sections. Each methylene group was found to contribute the same amount to the total collision cross section. Ions with cyclic structures were found to have smaller total collision cross sections compared to the corresponding noncyclic structures.

The observed absolute total collision cross sections of ions may be biased by instrumental parameters such as the pumping efficiency and the acceptance angle of the instrument. van Houte et al. [5] determined the absolute total collision cross sections of ions by measurements at different collision region lengths and extrapolation at zero target gas pressures. In that way, they avoided the difficulties associated with the measurement of the absolute pressure in the collision cell.

We have investigated the possibility of determination of the total collision cross sections on a relative basis. For the same mass spectrometer, the instrumental parameters are constant. Relative cross sections can be obtained for collisions of a precursor ion with a target gas and normalized to the collision cross section of a standard ion obtained under the same experimental conditions with the same target gas. Absolute cross sections are difficult to measure and probably not necessary for the purposes of the calculations of the relative sensitivities of selected precursors. The experimentally obtained relative total collision cross sections have been correlated empirically with known properties of the collision system. In that manner, it is possible to calculate the relative collision cross sections of selected ion structures without prior experimentation.

\section{Experimental}

A JEOL (Peabody, MA) JMS-AX505 double focusing sector mass spectrometer was used for the experiments. Collision-activated dissociation was performed at the first field-free region (FFR1) of the instrument. The conditions in the electron ionization source were: ion source temperature, $230{ }^{\circ} \mathrm{C}$; ion current, $300 \mu \mathrm{A}$; accelerating voltage, $3 \mathrm{kV}$; electron energy, $70 \mathrm{eV}$; resolution, 1500. Samples were introduced through a heated reservoir $\left(130^{\circ} \mathrm{C}\right)$. All samples were obtained from commercial sources and were used without further purification. Ultra high purity ( $>99.999 \%)$ helium (Liquid Carbonic Inc., Corunna, $\mathrm{ON}$ ) was used as target gas. A JEOL penning ionization gauge meter, located in the ionization source housing close to the exit of the collision cell, was used to measure the pressure that resulted from the admission of the target gas. It was not possible to measure the pressure inside the collision cell. Before the admission of the target gas, the intensity of the main beam was set to a maximum setting (100\%). The target gas was then admitted, which resulted in an attenuation of the main beam intensity. The pressure was recorded for replicate measurements at main beam attenuation levels between 10 and $90 \%$. The attenuation levels of the main beam intensity were monitored by using the oscilloscope or the data system of the mass spectrometer. No corrections were made in the pressure readings for the nature of the target gas. Following the initial tuning of the instrument, all experimental variables were unchanged for the duration of the day. $n$-Octane was analyzed at each experimental session. All collision cross sections were normalized to the collision cross section of $n$-octane (1.00). The reproducibility of the measurements was monitored for a period longer than 6 months.

\section{Results and Discussion}

The total collision cross section $\sigma_{\mathrm{c}}$ can be determined from the equation [6]

$$
I / I_{0}=\exp \left(-\sigma_{\mathrm{c}} n l\right)
$$

$I$ and $I_{0}$ are the intensities of the transmitted ion beam with and without the presence of the target gas, respectively, $n$ is the number density of the target gas, and $l$ is the collision cell length. The equation can be rearranged:

$$
\ln \left(I_{0} / I\right)=k \sigma_{\mathrm{c}} P
$$

$k$ is a constant and $P$ is the measured target gas pressure. A plot of $\ln \left(I_{0} / I\right)$ versus $P$ can thus lead to the collision cross section. This approach to determine the relative collision cross section of different precursor ions applies only to experiments done on the same mass spectrometer by using the same target molecule. The approach is demonstrated in Figure 1 for the evaluation of the relative cross section of $n$-pentane $(m / z$ 72). Similar linear plots with different slopes are obtained for the other organic precursors that collide with helium. The linearity of this plot was observed previously by Todd and McLafferty [6].

\section{Reproducibility}

The reproducibility of the approach has been monitored for a period of at least 6 months. Table 1 summarizes the percent standard deviations of the values determined for the $C_{5}$ to $C_{12}$ normal alkanes relative to the value of $n$-octane. Results are based on the average of at least three replicate measurements. Each 


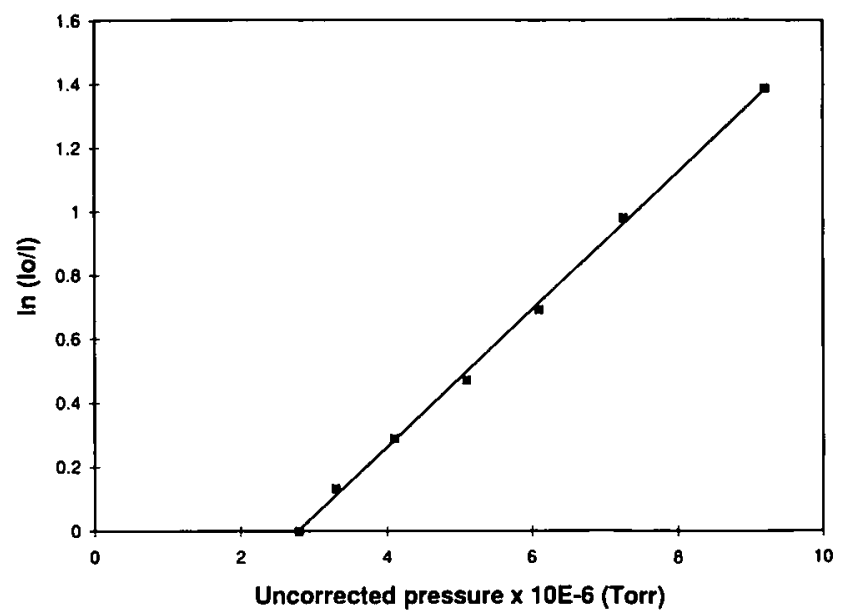

Figure 1. Typical determination of the relative collision cross section of organic ions. The slope of the plot is related to the total cross section of the collision of the precursor ion ( $n$-pentane, $\mathrm{m} / \mathrm{z}$ 72) with helium.

day, prior to the commencement of the experiments, the instrument was tuned for maximum sensitivity in accordance with standard mass spectrometric procedures. Tuning parameters could be very different over the period in which the measurements were made. Reproducibility is best, as expected, for measurements done within the same day (Table 1). The percent standard deviation for $n$-alkanes is less than $2 \%$ for the short term experiments. Reproducibility is worse ( $n$ pentane, 9\%) for the long term experiments (a 7-month period). This can be explained by possible long term changes in the ionization source due to performance of different experiments (chemical ionization, liquid chromatography, etc.). The medium and long term experiments revealed a slightly better reproducibility for the heavier ions. This may be because of the need for higher target gas pressures to attenuate the smaller ions compared to the pressures needed to attenuate the larger ions by the same fraction. For instance, to attenuate $n$-pentane $(\mathrm{m} / z \mathrm{z})$ to $50 \%$ of its original intensity one needs approximately 1.4 times the pressure needed to attenuate $n$-decane ( $m / z 142)$ to the same level. This means that the slopes of the smaller ions are obtained by using fewer data points, because there is an upper limit of pressure that the mass spectrometer will withstand. It could be that calculation of the slopes of the plots by using fewer data points results in less (medium and long term) reproducible results for the smaller ions. Overall, the long term reproducibilities have shown that when the same instrument and the same target gas are used, it is possible to determine the relative collision cross sections of selected precursor ions with an acceptable level of precision.

Small temperature changes $\left(20\right.$ to $30^{\circ} \mathrm{C}$ ) in the ionization source and sample introduction inlet did not produce measurable changes in the collision cross sections. The resolution of the instrument for the selection of the precursor was expected to have some effect on
Table 1. Reproducibility (\% standard deviation) of relative collision cross sections ${ }^{a}$

\begin{tabular}{lccc}
\hline Compound & Short term $^{\mathrm{b}}$ & Medium term $^{\mathrm{c}}$ & Long term $^{\mathrm{d}}$ \\
\hline \hline$n$-Pentane & 0.3 & 4.5 & 8.6 \\
$n$-Hexane & 0.5 & 4.0 & 3.8 \\
$n$-Heptane & 0.4 & 1.9 & 3.4 \\
$n$-Nonane & 1.6 & 2.0 & 2.7 \\
$n$-Decane & 0.2 & 1.1 & 0.8 \\
$n$-Undecane & 1.1 & 0.5 & 2.3 \\
$n$-Dodecane & 0.8 & 2.1 & 0.7 \\
\hline
\end{tabular}

All collision cross sections are normalized to the collision cross section of $n$-octane.

${ }^{b}$ Average of three replicate measurements conducted on the same day with the same instrumental tuning conditions.

cAverage of two sets of three replicate measurements made on different days over a 2 -week period with different instrumental tuning conditions

${ }^{d}$ Average of two sets of three replicate measurements made over a 7-month period with different instrumental tuning conditions.

the measurements; however, changes in the beta slit width did not greatly affect ion selection. Similarly, changes in the main and alpha slit widths affected only ion abundances. The resolution for precursor ion selection in the JEOL AX505 is estimated to be between 100 to 300 . This is due to the small size of the instrument and the limited accelerating voltage $(3 \mathrm{kV})$. The slit widths of the instrument were, therefore, tuned for a mass resolution of 1500 for all experiments.

\section{n-Alkanes}

The results obtained for $n$-alkanes are summarized in Table 2 . The values in parentheses represent the standard deviation of the measurements. The collision cross sections increase from 0.64 for $n$-pentane to 1.38 for $n$-dodecane. A linear relationship is obtained between $\sigma_{c}$ and the number of carbon atoms $n$ in the alkane:

$$
\sigma_{\mathrm{c}}(n)=0.105 n+0.137
$$

The coefficient of determination $\left(R^{2}\right)$ of the equation is 0.996. Each methylene group contributes 0.1 to the cross section. The $\sigma_{c}$ values of the $n$-alkanes are in good agreement with the values obtained by van Houte et al. [5]. Their results, normalized to the value of $n$-octane, are shown in Table 2 . It is worth noticing that these workers did not use helium as target gas and their results are the total collision cross sections that correspond to zero target pressure.

Relative molar sensitivities in 70-eV electron ionization experiments are directly related to the relative ionization cross sections of chemical structures [1]. Fitch and Sauter [7] developed an empirical scheme to calculate the total ionization cross sections $\left(\sigma_{i}\right)$ of organic molecules. Their method is based on the additivity of atomic ionization cross sections and requires only the chemical formula of a molecule for the calculation. A simple approach to determine the $\sigma_{c}$ values is to correlate the measured $\sigma_{c}$ values with known 
Table 2. Relative collision cross sections of $n$-alkanes ${ }^{a}$

\begin{tabular}{|c|c|c|c|c|}
\hline Compound & $\begin{array}{c}\text { Collision cross } \\
\text { section }^{\mathrm{b}} \sigma_{\mathrm{c}}\end{array}$ & $\begin{array}{l}\text { Collision cross } \\
\text { section }^{c} \sigma_{\mathrm{c}}\end{array}$ & $\begin{array}{c}\text { Ionization cross } \\
\text { section }^{\mathrm{d}} \sigma_{\mathrm{i}}\end{array}$ & $(n+M) / n$ \\
\hline$n$-Pentane & $0.64(0.03)$ & 0.68 & 0.65 & 0.65 \\
\hline$n$-Hexane & $0.77(0.03)$ & 0.78 & 0.76 & 0.76 \\
\hline$n$-Heptane & $0.86(0.02)$ & 0.86 & 0.88 & 0.88 \\
\hline$n$-Octane & $1.00(0.01)$ & 1.00 & 1.00 & 1.00 \\
\hline$n$-Nonane & $1.08(0.02)$ & 1.08 & 1.12 & 1.12 \\
\hline$n$-Decane & $1.20(0.01)$ & 1.15 & 1.24 & 1.23 \\
\hline$n$-Undecane & $1.27(0.01)$ & - & 1.35 & 1.35 \\
\hline$n$-Dodecane & $1.38(0.03)$ & - & 1.47 & 1.47 \\
\hline
\end{tabular}

${ }^{a}$ All collision cross sections are normalized to the collision cross section of $n$-octane.

${ }^{b}$ Measured relative collision cross sections. Values in parentheses denote the standard deviation of the measurements.

'Relative collision cross sections from van Houte, Koster, and van Thuijl (ref 5).

donization cross sections calculated via the scheme of Fitch and Sauter (ref 7 ).

properties of the collision system. The total ionization cross section $\left(\sigma_{\mathrm{i}}\right)$ is such a property that can be determined easily by using the approach of Fitch and Sauter. Another known value for a given collision system is the $(n+M) / n$ ratio. $M$ is the mass of the selected ion and $n$ is the mass of the target molecule. The $\sigma_{\mathrm{i}}$ and $(n+M) / n$ values for $n$-alkanes are shown in Table 2 . Coincidentally, these values are almost identical in the case of $n$-alkanes. This is not the case in general, as will be seen from the other structures in the study. In the simplest case, $\sigma_{\mathrm{c}}$ could be linearly related to $\sigma_{\mathrm{i}}$, based on the relative similarities between the molecule and ion structures (neglecting isomerization reactions, at a first approximation). Another reasonable hypothesis could expect a simple relationship between $\sigma_{c}$ and $(n+M) / n$, based on the fraction of precursor ion kinetic energy that is available to be converted into internal energy upon collision. Indeed, a linear relationship with $R^{2}=0.996$ is obtained between $\sigma_{c}$ and $\sigma_{i}$ for the $n$-alkanes. Similar good linearity is observed between $\sigma_{c}$ and $(n+M) / n$. However, poorer correlations are obtained when all other compounds are included, which indicates that more complex relationships exist between the various properties of the collision system.

\section{n-Alkylbenzenes}

The relative collision cross sections of $n$-alkylbenzenes (benzene to $n$-decylbenzene) increase linearly from the smaller to the larger structures (Table 3 ). The $\sigma_{c}$ values can be obtained from the number of carbon atoms $n$ in the alkylbenzene via the equation

$$
\sigma_{\mathrm{c}}=0.099 n-0.124
$$

The correlation coefficient for the $n$-alkylbenzenes equation is 0.993 . Each methylene group contributes 0.1 to $\sigma_{c}$ per carbon number. The methylene group contribution is thus the same in both $n$-alkanes and $n$-alkylbenzenes. However, the intercepts are different for the two chemical types. Smaller $\sigma_{\mathrm{c}}$ values are obtained for the alkylaromatic compounds than for the $n$-alkanes with the same carbon number. For example, toluene has a $\sigma_{\mathrm{c}}$ value of 0.55 compared to the $\sigma_{\mathrm{c}}$ of heptane, which is 0.86 . This may reflect the relative differences in the size and stabilities of the alkylbenzenes compared to the $n$-alkanes with the same carbon number. Good correlations are obtained between $\sigma_{c}$ versus $\sigma_{i}$ and $\sigma_{c}$ versus $(n+M) / n$ (coefficients of correlation are 0.993 and 0.994 respectively). The equation that relates $\sigma_{\mathrm{c}}$ with $\sigma_{\mathrm{i}}$ for $n$-alkanes is

$$
\sigma_{\mathrm{c}}=0.89 \sigma_{\mathrm{i}}+0.09
$$

For $n$-alkylbenzenes the equation is

$$
\sigma_{\mathrm{c}}=0.84 \sigma_{\mathrm{i}}+0.03
$$

The slope and intercept in the $n$-alkylbenzene equation are both smaller than those in the $n$-alkanes equation.

Table 3. Relative collision cross sections of n-alkylbenzenes

\begin{tabular}{lccc}
\hline Compound & $\begin{array}{c}\text { Collision cross } \\
\text { section }^{\mathrm{a}} \sigma_{\mathrm{c}}\end{array}$ & $\begin{array}{c}\text { lonization cross } \\
\text { section }^{\mathrm{b}} \sigma_{i}\end{array}$ & $(n+M) / n$ \\
\hline \hline Benzene & $0.43(0.01)$ & 0.53 & 0.69 \\
Toluene & $0.55(0.02)$ & 0.64 & 0.81 \\
Ethylbenzene & $0.68(0.02)$ & 0.76 & 0.93 \\
n-Propylbenzene & $0.78(0.01)$ & 0.88 & 1.05 \\
n-Butylbenzene & $0.91(0.02)$ & 1.00 & 1.17 \\
n-Pentylbenzene & $1.00(0.03)$ & 1.12 & 1.29 \\
n-Hexylbenzene & $1.11(0.01)$ & 1.23 & 1.41 \\
n-Heptylbenzene & $1.17(0.02)$ & 1.35 & 1.53 \\
$n$-Octylbenzene & $1.25(0.02)$ & 1.47 & 1.64 \\
n-Nonylbenzene & $1.35(0.05)$ & 1.59 & 1.76 \\
n-Decylbenzene & $1.44(0.01)$ & 1.70 & 1.88 \\
\hline
\end{tabular}

\footnotetext{
${ }^{9}$ Measured relative collision cross sections. Values in parentheses denote the standard deviation of the measurements.

b lonization cross sections calculated via the scheme of Fitch and Sauter (ref 7).
} 
The $n$-alkanes equation for the calculation of $\sigma_{\mathrm{c}}$ from the $(n+M) / n$ values is

$$
\sigma_{\mathrm{c}}=0.89((n+M) / n)+0.09
$$

The corresponding $n$-alkylbenzenes equation is

$$
\sigma_{\mathrm{c}}=0.84((n+M) / n)-0.11
$$

The slopes of eqs 7 and 8 are the same as the slopes in eqs 5 and 6 . These equations demonstrate the linear dependence of $\sigma_{\mathrm{c}}$ on both $\sigma_{\mathrm{i}}$ and $(n+M) / n$. However, eqs 5 and 7 can be used only for $n$-alkanes and eqs 6 and 8 for $n$-alkylbenzenes. These simple relationships do not permit the calculation of $\sigma_{\mathrm{c}}$ for a precursor ion that does not belong to these two chemical types.

\section{Other Compounds}

The collision cross sections of some aromatic sulfur and chlorinated compounds are presented in Table 4. Thiophene has a small $\sigma_{\mathrm{c}}$ value $(0.41)$, which is close to the value of benzene (0.43). Alkyl substitution on thiophene increases the collision cross section. 2methylthiophene has a $\sigma_{c}$ value of 0.50 and tert-butylthiophene has $\sigma_{\mathrm{c}}=0.84$. Collision cross sections increase with molecular size.

The three dichlorobenzene isomers (Table 4) have an average $\sigma_{\mathrm{c}}$ value of 0.55 , which is higher than benzene (0.43). This implies that the effective contribution of each chlorine atom on benzene is less than the contribution of a methylene group. The $\sigma_{\mathrm{c}}$ value of ethylbenzene $(0.68)$ is higher than that of the dichlorobenzenes (0.55). The corresponding $\sigma_{\mathrm{i}}$ values for ethylbenzene and dichlorobenzene are very similar (0.76 and 0.79$)$. On the contrary, the $(n+M) / n$ values are different for ethylbenzene and dichlorobenzene (0.93 and 1.27). For chemical structures with similar

Table 4. Relative collision cross sections of thiophenes and dichlorobenzenes

\begin{tabular}{lccc}
\hline Compound & $\begin{array}{c}\text { Collision } \\
\text { cross } \\
\text { section }^{\mathrm{a}} \sigma_{\mathrm{c}}\end{array}$ & $\begin{array}{c}\text { lonization } \\
\text { cross } \\
\text { section }^{\mathrm{b}} \sigma_{\mathrm{i}}\end{array}$ & $(n+M) / n$ \\
\hline \hline Thiophene & $0.41(0.02)$ & 0.51 & 0.75 \\
2-Methylthiophene & $0.50(0.02)$ & 0.62 & 0.86 \\
3-Methylthiophene & $0.48(0.02)$ & 0.62 & 0.86 \\
2,5-Dimethylthiophene & $0.60(0.01)$ & 0.74 & 0.98 \\
2-Ethylthiophene & $0.64(0.01)$ & 0.74 & 0.98 \\
tert-Butylthiophene & $0.84(0.03)$ & 0.98 & 1.22 \\
& & & \\
1.2-Dichlorobenzene & $0.55(0.01)$ & 0.79 & 1.27 \\
1.3-Dichlorobenzene & $0.54(0.01)$ & 0.79 & 1.27 \\
1,4-Dichlorobenzene & $0.56(0.01)$ & 0.79 & 1.27 \\
\hline
\end{tabular}

Measured relative collision cross sections. Values in parentheses denote the standard deviation of the measurements.

'Ionization cross sections calculated via the scheme of Fitch and Sauter (ref 7). total ionization cross sections, the masses of the collision system influence the collision cross sections.

Table 5 contains the collision cross sections of some sulfides and disulfides. The table also displays the data of $\sigma_{\mathrm{i}}$ and $(n+M) / n$ for these compounds. The $\sigma_{\mathrm{c}}$ values increase from the smaller to the larger sulfide and disulfide compounds. The disulfide collision cross sections are larger than the corresponding values of the sulfides. By comparison of the methyl, propyl, and butyl sulfide and disulfide compounds, the sulfur atom is deduced to contribute an average of 0.12 to the $\sigma_{c}$ of the linear sulfur compounds. Simple addition of the methylene and sulfur $\sigma_{c}$ values approximately leads to the measured $\sigma_{c}$ values for the linear sulfur compounds. The additivity rule also holds true for the thiophenes (Table 4) when 0.1 is added for each $\mathrm{CH}_{2(3)}$ group to the $\sigma_{\mathrm{c}}=0.41$ of the thiophene nucleus. The $\mathrm{CH}$ group in the aromatic nucleus contributes a smaller amount to $\sigma_{\mathrm{c}}$ compared to the contribution of the methylene group in the linear compounds.

The relative collision cross section of some additional $\mathrm{C}_{x} \mathrm{H}_{y} \mathrm{O}_{z} \mathrm{~N}_{t} \mathrm{Cl}_{s} \mathrm{Br}_{p}$ compounds are shown in Table 6. The average standard deviation in the measurements made on different days over a 3-week period was $3.51 \%$.

\section{Additivity Approach}

A statistical approach similar to that undertaken by Fitch and Sauter [7] is possible. The equation obtained after a linear regression that used the 53 measured total collision cross sections is

$$
\begin{aligned}
\sigma_{\mathrm{c}}= & 0.0888+0.0328 n_{\mathrm{C}}+0.0333 n_{\mathrm{H}} \\
& +0.0273 n_{\mathrm{O}}-0.0116 n_{\mathrm{N}}+0.0414 n_{\mathrm{S}} \\
& +0.0663 n_{\mathrm{Cl}}+0.1055 n_{\mathrm{Br}}
\end{aligned}
$$

Table 5. Relative collision cross sections of sulfides and disulfides

\begin{tabular}{lccc}
\hline Compound & $\begin{array}{c}\text { Collision } \\
\text { cross } \\
\text { section }\end{array}$ & $\begin{array}{c}\text { lonization } \\
\text { cross }\end{array}$ & \\
\hline \hline Methyl sulfide & $0.36(0.02)$ & 0.45 & 0.56 \\
Ethyl sulfide & $0.57(0.03)$ & 0.68 & 0.80 \\
Ethyl methyl sulfide & $0.46(0.02)$ & 0.57 & 0.68 \\
Propyl sulfide & $0.76(0.01)$ & 0.92 & 1.03 \\
Butyl sulfide & $0.94(0.02)$ & 1.15 & 1.27 \\
Hexyl sulfide & $1.31(0.02)$ & 1.62 & 1.75 \\
Ethyl phenyl sulfide & $0.72(0.01)$ & 0.92 & 1.20 \\
Phenyl sulfide & $0.87(0.02)$ & 1.15 & 1.61 \\
Methyl disulfide & $0.47(0.01)$ & 0.60 & 0.83 \\
Propyl ethyl disulfide & $0.69(0.03)$ & 0.96 & 1.19 \\
Propyl disulfide & $0.89(0.01)$ & 1.07 & 1.30 \\
Butyl disulfide & $1.06(0.01)$ & 1.31 & 1.54 \\
\hline
\end{tabular}

\footnotetext{
"Measured relative collision cross sections. Values in parentheses denote the standard deviation of the measurements.

Ionization cross sections calculated via the scheme of Fitch and Sauter (ref 7).
} 
Table 6. Relative collision cross sections of various $\mathrm{C}_{x} \mathrm{H}_{y} \mathrm{O}_{z} \mathrm{~N}_{i} \mathrm{Cl}_{s} \mathrm{Br}_{p}$ compounds

\begin{tabular}{lccc}
\hline Compound & $\begin{array}{c}\text { Collision } \\
\text { cross } \\
\text { section }\end{array} \sigma_{\mathrm{c}}$ & $\begin{array}{c}\text { lonization } \\
\text { cross } \\
\text { section }^{\mathrm{b}} \sigma_{\mathbf{i}}\end{array}$ & $(n+M) / n$ \\
\hline \hline Propanol & 0.49 & 0.46 & 0.54 \\
Acetone & 0.41 & 0.40 & 0.53 \\
Methyl ethyl ketone & 0.51 & 0.52 & 0.64 \\
Phenol & 0.50 & 0.57 & 0.83 \\
Pyridine & 0.42 & 0.49 & 0.70 \\
Quinoline & 0.61 & 0.78 & 1.13 \\
Aniline & 0.52 & 0.61 & 0.82 \\
Diethylamine & 0.62 & 0.61 & 0.65 \\
Isopropylamine & 0.40 & 0.49 & 0.53 \\
o-Bromophenol & 0.58 & 0.75 & 1.49 \\
$p$-Bromophenol & 0.59 & 0.75 & 1.49 \\
Chlorobenzene & 0.53 & 0.66 & 0.98 \\
\hline
\end{tabular}

${ }^{a}$ Measured relative collision cross sections. The average standard deviation of the measurements made on diferent days over a 3-week period was $3.51 \%$.

b Ionization cross sections calculated via the scheme of Fitch and Sauter (ref 7 ).

The correlation coefficient is $0.989 . n_{i}$ is the number of atoms, with $i=$ carbon, hydrogen, oxygen, nitrogen, sulfur, chlorine, and bromine. The validity of this equation is restricted within the results of the present study. A much larger data base is required for a thorough statistical study. This kind of analysis does not account for the position of the atom in the chemical structure and does not allow for the calculation of the collision cross sections of isomeric structures. However, eq 9 allows the collision cross sections of the precursors ions that collide with helium to be calculated and requires only knowledge of the chemical formula of the precursor. The plot between the measured and predicted collision cross sections, via eq 9 , is shown in Figure 2. The predicted $\sigma_{\mathrm{c}}$ values differ from the measured values by an average of $3.5 \%$.

$\sigma_{c}$ versus $\left[\sigma_{i}^{2}(n /(n+M))\right]$

Although good correlations exist between $\sigma_{\mathrm{c}}$ and $\sigma_{\mathrm{i}}$ for the individual chemical types ( $n$-alkanes, eq 5; $n$-alkylbenzenes, eq 6), a poor correlation is obtained when all collision cross sections are included. Figure 3 displays the plot of $\sigma_{\mathrm{c}}$ versus $\sigma_{\mathrm{i}}$ for the 53 ions. The coefficient of determination $R^{2}$ is 0.942 . Therefore, collision cross sections cannot be obtained directly from the ionization cross sections of the structure.

A better correlation $\left(R^{2}=0.975\right)$ has been found empirically to exist between $\sigma_{\mathrm{c}}$ and $\left[\sigma_{\mathrm{i}}^{2}(n /(n+M))\right]$ :

$$
\sigma_{\mathrm{c}}=0.137+0.839\left[\sigma_{\mathrm{i}}^{2}(n /(n+M))\right]
$$

The results are shown in Figure 4. Incorporation of the $n /(n+M)$ term improves the correlation between $\sigma_{\mathrm{c}}$ and $\sigma_{\mathrm{i}}$. Equation 10 permits the calculation of the $\sigma_{\mathrm{c}}$

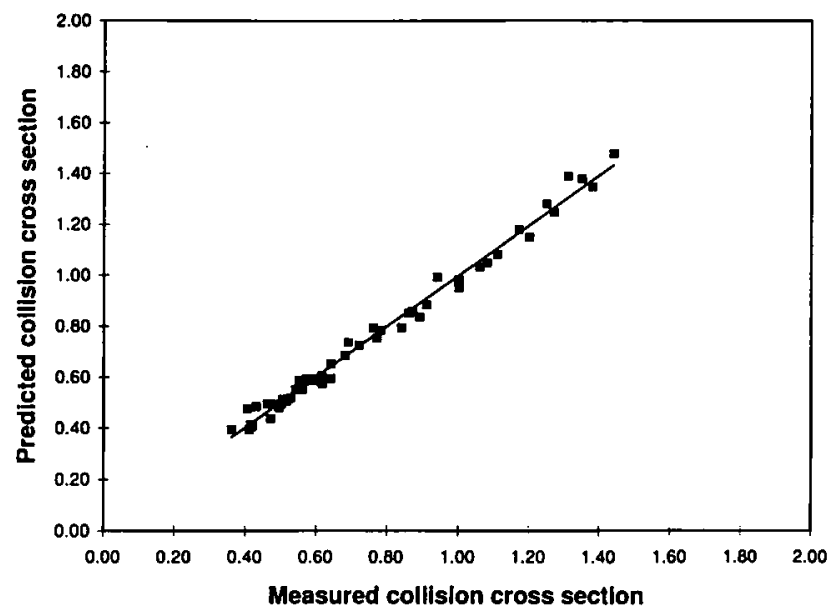

Figure 2. Predicted versus measured relative collision cross section. Equation 9 is used for the calculations. The coefficient of determination is 0.989 . All cross sections are relative to $n$-octane (1.00).

values based on the known values of $\sigma_{i}$ and the masses of the collision partners. The average difference between the results obtained via eq 10 and the experimental $\sigma_{\mathrm{c}}$ values is $5.5 \%$.

\section{Theoretical Considerations}

The general collision theory of ion-molecule reactions [8-11] was designed for capture collisions and does not apply directly to the high energy collision experiments of the present study. However, previous studies $[1,2,13,14]$ established good correlations between the ionization cross sections and the polarizabilities of the molecules. Therefore, the relationship between the experimental collision cross sections and the corresponding molecular polarizabilities was investigated. A very poor correlation $\left(R^{2}=0.881\right)$ is obtained in Tables $1-5$, between $\sigma_{\mathrm{c}}$ and polarizability $\alpha$ for the 40 precursor

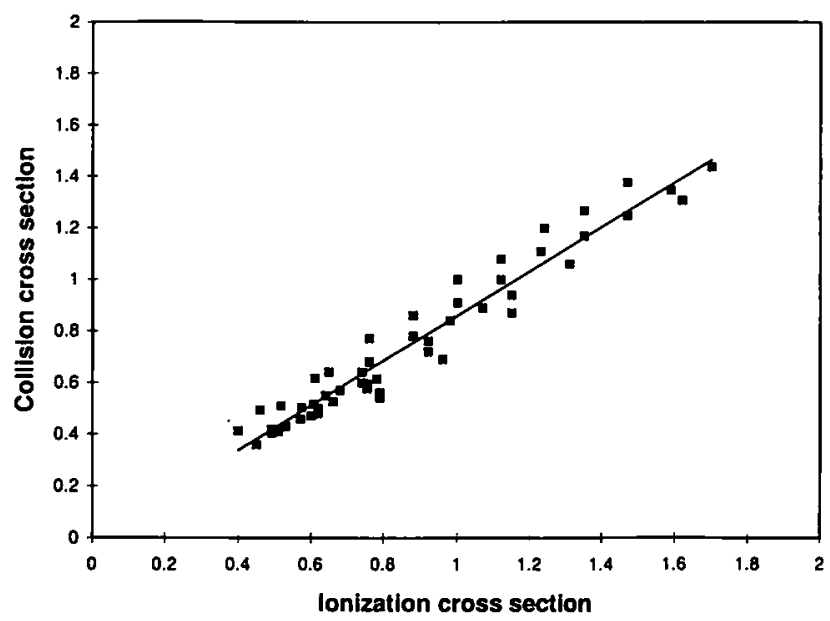

Figure 3. Measured collision cross section versus calculated ionization cross section. Ionization cross sections are calculated via the scheme of Fitch and Sauter (ref 7). 


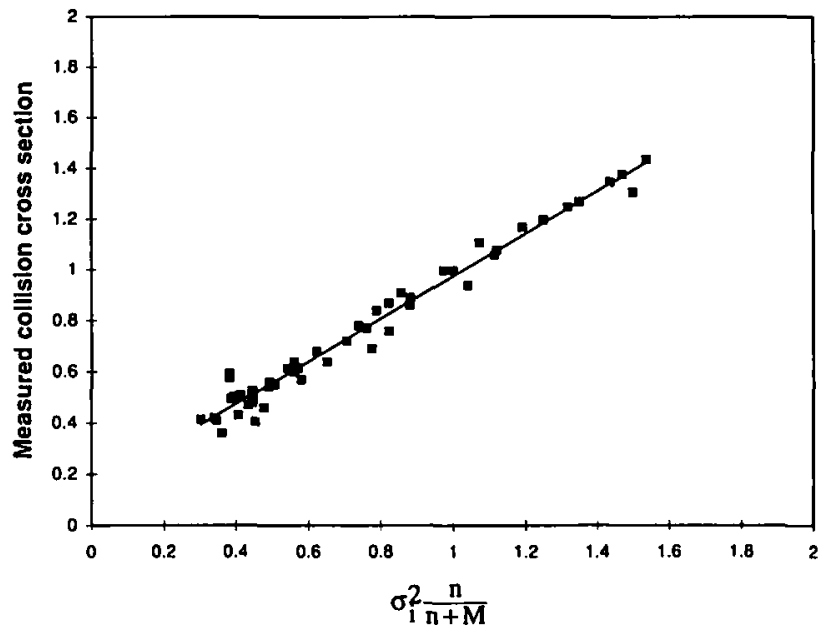

Figure 4. Measured relative collision cross section $\sigma_{c}$ versus $\sigma_{i}^{2}(n /(n+M))$.

ions. However, good correlations between $\sigma_{\mathrm{c}}$ and $\alpha$ are found for the individual chemical series. The $R^{2}$ for $n$-alkanes is 0.996 and for $n$-alkylbenzenes is 0.993 . The polarizability values were determined with the method of Miller and Savchik [12]. Similar to the correlations of $\sigma_{\mathrm{i}}$ with polarizability, $\sigma_{\mathrm{c}}$ correlates well with the $\alpha$ values for an individual chemical series, but different equations are needed to calculate the values of different series.

The high energy collisions in the present study involve short range repulsive forces and can be treated as collisions between rigid spheres [15]. The collision cross section between two spheres, in the hard-sphere model, is given by

$$
\sigma_{\mathrm{c}}=\int_{0}^{d} 2 \pi b d b=\pi d^{2}
$$

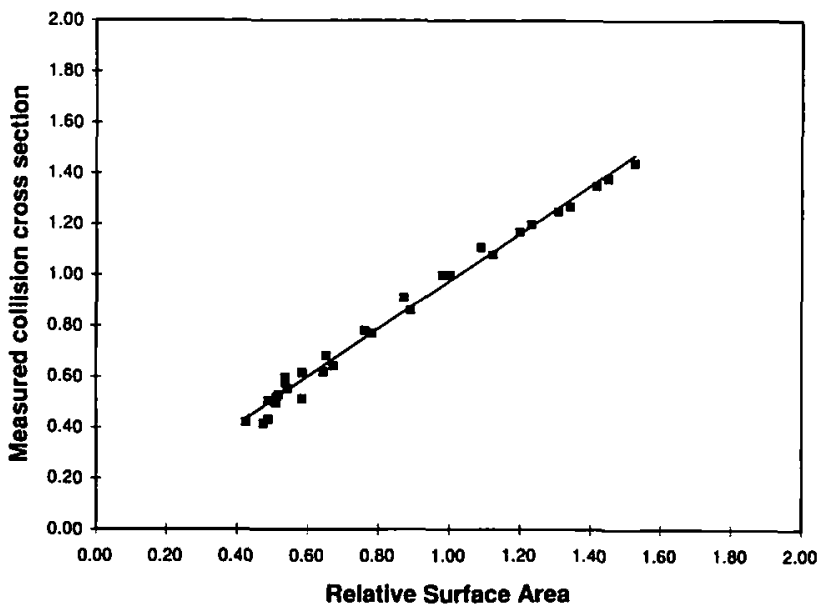

Figure 5. Measured relative collision cross section $\sigma_{\mathrm{c}}$ versus relative molecular surface area. Molecular surface areas were calculated according to Bondi (ref 16). where $d$ is the sum of the radii of the two spheres and $b$ is the distance of the closest approach between the two spheres. Equation 11 shows that $\sigma_{\mathrm{c}}$ is related to the surface area of the collision system. Figure 5 displays the plot between the measured collision cross sections of the ions and the corresponding relative molecular surface areas calculated according to Bondi [16]. Unfortunately, the surface area of the sulfur compounds could not be evaluated because the sulfur group contributions to the surface area were not available in the article by Bondi. However, a good correlation $\left(R^{2}=0.989\right)$ is obtained between the observed $\sigma_{\mathrm{c}}$ values and the nonsulfur molecular surface areas $A$. The equation is

$$
\sigma_{\mathrm{c}}=0.035+0.940 \mathrm{~A}
$$

The average difference between the experimental values and those obtained via eq 12 is $4.0 \%$. Further investigation that neglects the geometry of structures and considers an additive relationship of the van der Waals radii $\rho_{\mathrm{i}}$ [16] for the 53 ions results in the equation

$$
\sigma_{\mathrm{c}}=0.097+0.849 \sum \rho_{\mathrm{i}}
$$

$\sum \rho_{\mathrm{i}}$ is a simple summation of the atomic van der Waals radii in the ion (Figure 6). The $R^{2}$ of eq 13 is 0.982 . The average difference between the observed $\sigma_{\mathrm{c}}$ values and those obtained using eq 13 is $5.2 \%$. Both eqs 12 and 13 indicate good linear relationships between the molecular area and the collision cross sections of the ions, which are in agreement with eq 11.

\section{Ion Kinetic Energy and Nature of Target Gas}

The effect of the ion kinetic energy on the relative collision cross sections has been investigated with experiments at 1,2 , and $3 \mathrm{keV}$. The results obtained for several organic ions colliding with helium are summa-

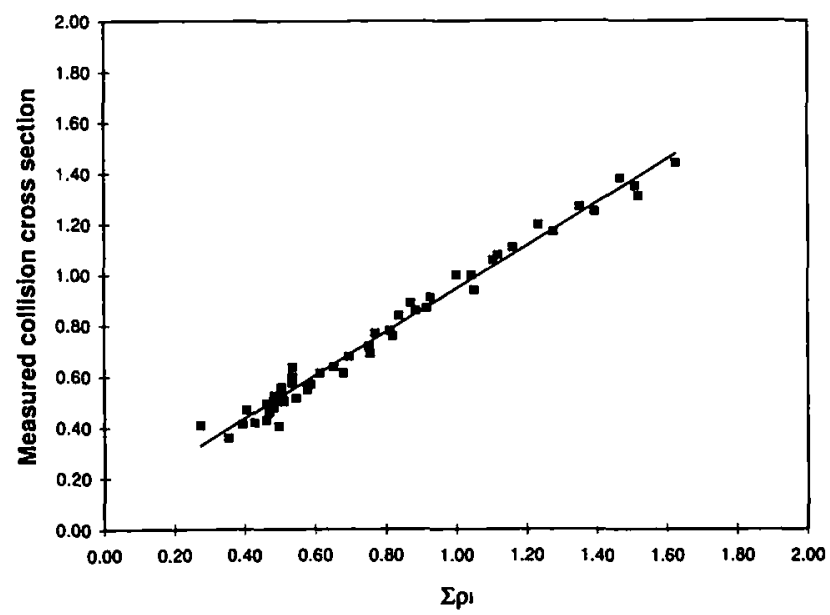

Figure 6. Measured relative collision cross section $\sigma_{\mathrm{c}}$ versus the relative sum of the atomic van der Waals radii $\left(\sum \rho_{i}\right)$. 
rized in Table 7. Slightly different results are obtained for the experiments at the three kinetic energies. The average standard deviation of the measurements at the three kinetic energies is $6.99 \%$, which is higher than the average long term repeatability of the measurements at a single ion kinetic energy regime (3.5\%). It is not possible at this stage to conclude if the observed differences are due to larger experimental errors associated with the tuning of the instrument or due to the differences in the ion kinetic energies.

The effect of the target gas nature has been examined by using helium, nitrogen, and argon as collision gases (Table 8). The average standard deviation of the measurements with the three gases is $4.25 \%$, which is close to the average long term repeatability $(3.5 \%)$ by using helium at $3 \mathrm{keV}$. These results indicate that the relative total collision cross sections depend primarily on the size of the precursor ion and less on the nature of the target gas. This is to be expected because collision cross sections are normalized to the value of a common precursor ion that collides with the same target molecule. Although absolute total collision cross sections are generally different for collisions with different targets, the normalization step removes the dependence of the relative collision cross sections on the nature of the target gas. The main objective of the present work was to determine the relative collision cross sections by using helium as a collision gas at $3-\mathrm{keV}$ ion kinetic energy, based on the needs and capabilities of our laboratory. The limited results, how-

Table 7. The effect of ion kinetic energy: relative collision cross sections of various $\mathrm{C}_{x} \mathrm{H}_{y} \mathrm{O}_{z} \mathrm{~N}_{t} \mathrm{~S}_{r} \mathrm{Cl}_{s} \mathrm{Br}_{p}$ compounds acquired at different ion kinetic energies with helium as collision gas

\begin{tabular}{lcccc}
\hline & \multicolumn{4}{c}{ Collision cross section ${ }^{\mathrm{a}} \sigma_{\mathrm{c}}$} \\
\cline { 2 - 5 } Compound & $3 \mathrm{keV}$ & $2 \mathrm{keV}$ & $1 \mathrm{keV}$ & Average \\
\hline \hline$n$-Heptane & 0.86 & 0.96 & 0.92 & $0.91(0.05)$ \\
$n$-Octane & 1.00 & 1.00 & 1.00 & $1.00(0.00)$ \\
$n$-Nonane & 1.08 & 1.12 & 1.03 & $1.07(0.05)$ \\
$n$-Decane & 1.20 & 1.23 & 1.18 & $1.20(0.02)$ \\
Thiophene & 0.41 & 0.44 & 0.44 & $0.43(0.02)$ \\
2-Methylthiophene & 0.50 & 0.57 & 0.53 & $0.53(0.03)$ \\
Propanol & 0.49 & 0.58 & 0.54 & $0.54(0.05)$ \\
Acetone & 0.41 & 0.50 & 0.46 & $0.46(0.04)$ \\
Methyl ethyl ketone & 0.51 & 0.62 & 0.58 & $0.57(0.06)$ \\
Phenol & 0.50 & 0.59 & 0.57 & $0.56(0.05)$ \\
Pyridine & 0.42 & 0.50 & 0.47 & $0.47(0.04)$ \\
Quinoline & 0.61 & 0.70 & 0.76 & $0.69(0.07)$ \\
Aniline & 0.52 & 0.61 & 0.58 & $0.57(0.05)$ \\
Diethylamine & 0.62 & 0.74 & 0.66 & $0.67(0.06)$ \\
Isopropylamine & 0.40 & 0.49 & 0.46 & $0.45(0.04)$ \\
o-Bromophenol & 0.58 & 0.63 & 0.57 & $0.59(0.03)$ \\
-Bromophenol & 0.59 & 0.68 & 0.65 & $0.64(0.04)$ \\
Chlorobenzene & 0.53 & 0.61 & 0.60 & $0.58(0.05)$ \\
\hline a & & & &
\end{tabular}

Measured relative collision cross sections. The average standard deviation of the measurements made at the three kinetic energies is $6.99 \%$.
Table 8. The effect of target gas: relative collision cross sections of various compounds acquired with different target gases

\begin{tabular}{lcccc}
\hline & \multicolumn{4}{c}{ Collision cross section ${ }^{\mathrm{a}} \sigma_{\mathrm{c}}$} \\
\cline { 2 - 5 } Compound & Helium & Nitrogen & Argon & Average \\
\hline \hline$n$-Heptane & 0.86 & 0.85 & 0.93 & $0.88(0.04)$ \\
$n$-Octane & 1.00 & 1.00 & 1.00 & $1.00(0.00)$ \\
$n$-Nonane & 1.08 & 1.04 & 1.08 & $1.07(0.02)$ \\
$n$-Decane & 1.20 & 1.14 & 1.13 & $1.15(0.04)$ \\
$n$-Undecane & 1.27 & 1.26 & 1.22 & $1.25(0.03)$ \\
Chlorobenzene & 0.53 & 0.59 & 0.63 & $0.58(0.05)$ \\
Quinoline & 0.61 & 0.72 & 0.65 & $0.66(0.06)$ \\
\hline
\end{tabular}

${ }^{\theta}$ Measured relative collision cross sections. The average standard deviation of the measurements made with the three gases is $4.25 \%$. The ion kinetic energy is $3 \mathrm{keV}$.

ever, obtained at different kinetic energies and different collision gases, suggest that the same approach can be applied with other gases and ion kinetic energy regimes. A larger study that involves instruments in different labs will reveal the generality of the approach.

\section{Isomers}

Previous studies have reported different collision cross sections for different isomeric structures $[4,5]$. The present study investigated a limited number of isomers. The $\sigma_{\mathrm{c}}$ values obtained for the various isomers are different, but the difference is not significant compared to the experimental error. 2- and 3-methyl thiophene have almost the same $\sigma_{c}$ values (Table 4). 2-ethylthiophene $(0.60)$ has a slightly smaller $\sigma_{c}$ value than 2,5-dimethylthiophene (0.64). The $\sigma_{c}$ values of the dichlorobenzenes are very similar (Table 4). The same is true for the ortho- and para-bromophenol isomers in Table 6. In the present experiments, selection of a precursor ion may involve the collisional activation of a mixture of possible ion structures. The reported $\sigma_{c}$ values represent the average collision cross section from the contributions of all selected isomeric structures at the same nominal mass. Better correlations may be obtained for systems with known isomeric structure distributions. Formation of precursor ions at different ionization electron energies can provide additional insight to the relationships between the nature of the chemical structure and its collision cross section with a neutral target molecule $[17,18]$.

\section{Conclusion}

The relative total cross sections of selected organic precursor ions that collide with helium have been obtained. Relative collision cross sections are simpler to obtain than absolute collision cross sections derived from direct collision cell pressure measurements or from extrapolations to zero target gas pressure. Experiments were performed with the same instrument un- 
der the same experimental conditions (electron energy, ion current, accelerating voltage, source temperature, and resolution). Relative collision cross sections obtained with an instrument of different geometry or under different experimental conditions may produce different $\sigma_{c}$ values. However, the approach of the present study should be valid for the determination of relative $\sigma_{c}$ values, which lead to quantitative relationships between precursor ions with different chemical structures, for any sector mass spectrometer.

Relative collision cross sections with helium as the target gas were found to increase with molecular size. Although good relationships were established between $\sigma_{c}$ and $\sigma_{i}$, and $\sigma_{c}$ and $\alpha$, multiple equations are required for the different chemical types ( $n$-alkanes, $n$-alkylbenzenes, etc.). A statistical approach that requires only the chemical formula of the precursor ion has allowed the prediction of the $\sigma_{c}$ values with an average error of $3.5 \%$. The empirical relationship $\left[\sigma_{i}^{2}(n /(n+M))\right]$, which involves the ionization cross section of the precursor structure and the masses of the collision system, also predicted the $\sigma_{\mathrm{c}}$ values with an average error of $5.5 \%$. A linear relationship was obtained between the collision cross section and the molecular surface area. Similarly, a good correlation was established with the sum of the atomic van der Waals radii. These results are in agreement with the hard-sphere collision theory that predicts that the collision cross section is a measure of the surface area of the collision system. Quantum mechanical potential interaction calculations between the moving precursor ion structure and the stationary neutral target may provide a better understanding of the collision process. Experiments at different electron ionization energies that form different distributions of precursor ion isomers may also help to establish better relationships between ion structures and their collision cross sections.

\section{References}

1. Allgood, C.; Orlando, R.; Munson, B. J. Am. Soc. Mass. Spectrom. 1990, 1, 397.

2. Bartmess, J. E.; Georgiadis, R. M. Vacuum 1983, 33, 149.

3. Laramee, J. A.; Cameron, D.; Cooks, R. G. J. Am. Chem. Soc. 1981, 103, 12.

4. van Tilborg, M. W. E. M.; van Thuijl, J. Org. Mass Spectrom. 1984, 19, 217.

5. van Houte, J. J.; de Koster, C. G.; van Thuijl, J. Int. J. Mass. Spectrom. Ion Processes 1992, 115, 173

6. Todd, P. J.; McLafferty, F. W. Int. J. Mass Spectrom. Ion Phys. $1981,38,371$.

7. Fitch, W. L.; Sauter, A. D. Anal. Chem. 1983, 55, 832.

8. Gioumousis, G.; Stevenson, D. P. J. Chem. Phys. 1958, 29, 294.

9. Field, F. H.; Franklin, J. L.; Lampe, F. W. J. Am. Chem. Soc. 1957, 79, 2419.

10. Su, T.; Bowers, M. T. J. Chem. Phys. 1973, 58, 3027.

11. Su, T.; Su, E. C. F.; Bowers, M. T. J. Chem. Phys. 1978, 69, 2243.

12. Miller, K. J.; Savchik, J. A. J. Am. Chem. Soc. 1979, 101, 7206.

13. Harrison, A. G.; Jones, E. G.; Gupta, S. K.; Nagy, G. P. Can. I. Chem. 1966, 44, 1967.

14. Beran, J. A.; Kevan, L. J. Phys. Chem. 1969, 73, 3866.

15. Levine, R. D.; Bernstein, R. B. Molecular Reaction Dynamics and Chemical Reactivity; Oxford University Press: New York, 1987; p 26.

16. Bondi, A. I. Phys. Chem. 1964, 68, 441.

17. Holmes, J. L. Org. Mass Spectrom. 1985, 20, 169.

18. van Houte, J. J.; van Thuijl, J. Int. J. Mass. Spectrom. Ion Processes 1994, 113, 121. 